\title{
Future of Biosimilar Products Packaging in Microgravity
}

\author{
Anupam Chanda* \\ Alembic Pharmaceuticals Limited and Indian Institute of Packaging Mumbai, India \\ *Corresponding Author: Anupam Chanda, Alembic Pharmaceuticals Limited and Indian Institute of Packaging Mumbai, India.
}

Received: October 21, 2019; Published: November 05, 2019

DOI: 10.31080/ASPS.2019.03.0433

\section{Introduction}

We all are well acquainted about criticality of Biosimilar products in different conditions during production and stability studies. Situation is more critical for those are especially highly viscous. Compatibility with primary packaging materials and selection of patient's friendly packaging device. We are lucky enough to deal this product in Earth condition and it's more challenging when deal in Microgravity environment like international space station, Moon , Mars and other planets where gravity, temperature and radiation levels are totally different. Product stability is the biggest challenge. We can't allow Astronauts and space tourist without proper treatment. Being a Biosimilar products Packaging material and device innovation scientist I ready don't know, how many years will take space will attain the similar kind of environment like Earth. Our Aim is to meet the present expectations.

\section{Purpose and special role}

- Innovation of most compatible primary packaging materials and device most suitable for microgravity environment.

- To ensure stability studies are carried out in line with microgravity environment.

- Secure life of Astronauts and space tourists.

- Possibility to establish fully operational testing laboratory in microgravity.

Few options, most suitable for microgravity

Long term exposure on different type of Pharmaceutical doses form especially different type of radiation exposure is most critical and rare thorough study has done. This has been observed solid dose is more stable compare to injectable and ophthalmic. Future research work would be more data oriented with facts and figures and mitigate the space radiation risk for future space expeditions.

Few imaginary Packaging designs for Biosimilar Products in Microgravity those will convert to reality one day:

Different packaging options suitable for Microgravity Environment

\begin{tabular}{|l|l|l|}
\hline $\begin{array}{c}\text { Type of } \\
\text { Product }\end{array}$ & $\begin{array}{c}\text { Recommended Primary } \\
\text { packaging Material }\end{array}$ & \multicolumn{1}{c|}{ Remark } \\
\hline Injectable & $\begin{array}{l}\text { Gold Lacquered Glass } \\
\text { vial is the most suit- } \\
\text { able to protect high } \\
\text { radiation } \\
\text { Compare to Rubber } \\
\text { stopper, PP stopper is } \\
\text { suitable in micro- } \\
\text { gravity. }\end{array}$ \\
\hline $\begin{array}{l}\text { Eye/Ear } \\
\text { Drop }\end{array}$ & $\begin{array}{l}\text { Gold Lacquered Glass } \\
\text { vial with screw type } \\
\text { neck is recommend- } \\
\text { ed for eye drop. } \\
\text { PP/PVC /Rubber+ } \\
\text { Aluminium Cap }\end{array}$ \\
\hline Inhalers & & $\begin{array}{l}\text { Common problem } \\
\text { most of the Astro- } \\
\text { nauts are facing, Ast- } \\
\text { hama problem, most } \\
\text { suitable packaging } \\
\text { is outside gold foil } \\
\text { coated container. } \\
\text { Helps to protect high } \\
\text { radiation. }\end{array}$ \\
\hline
\end{tabular}

Table 1

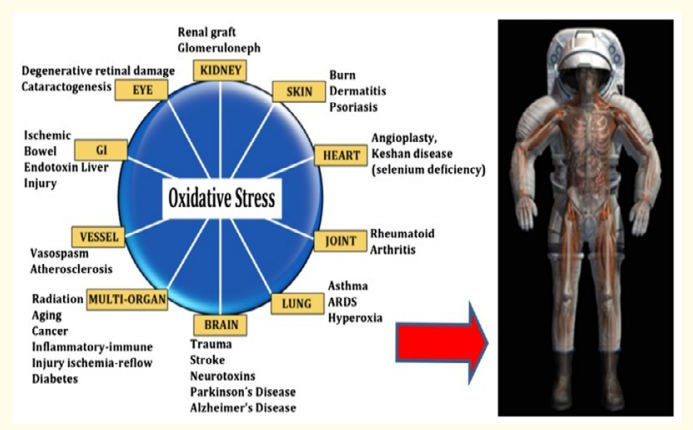

Figure 1 


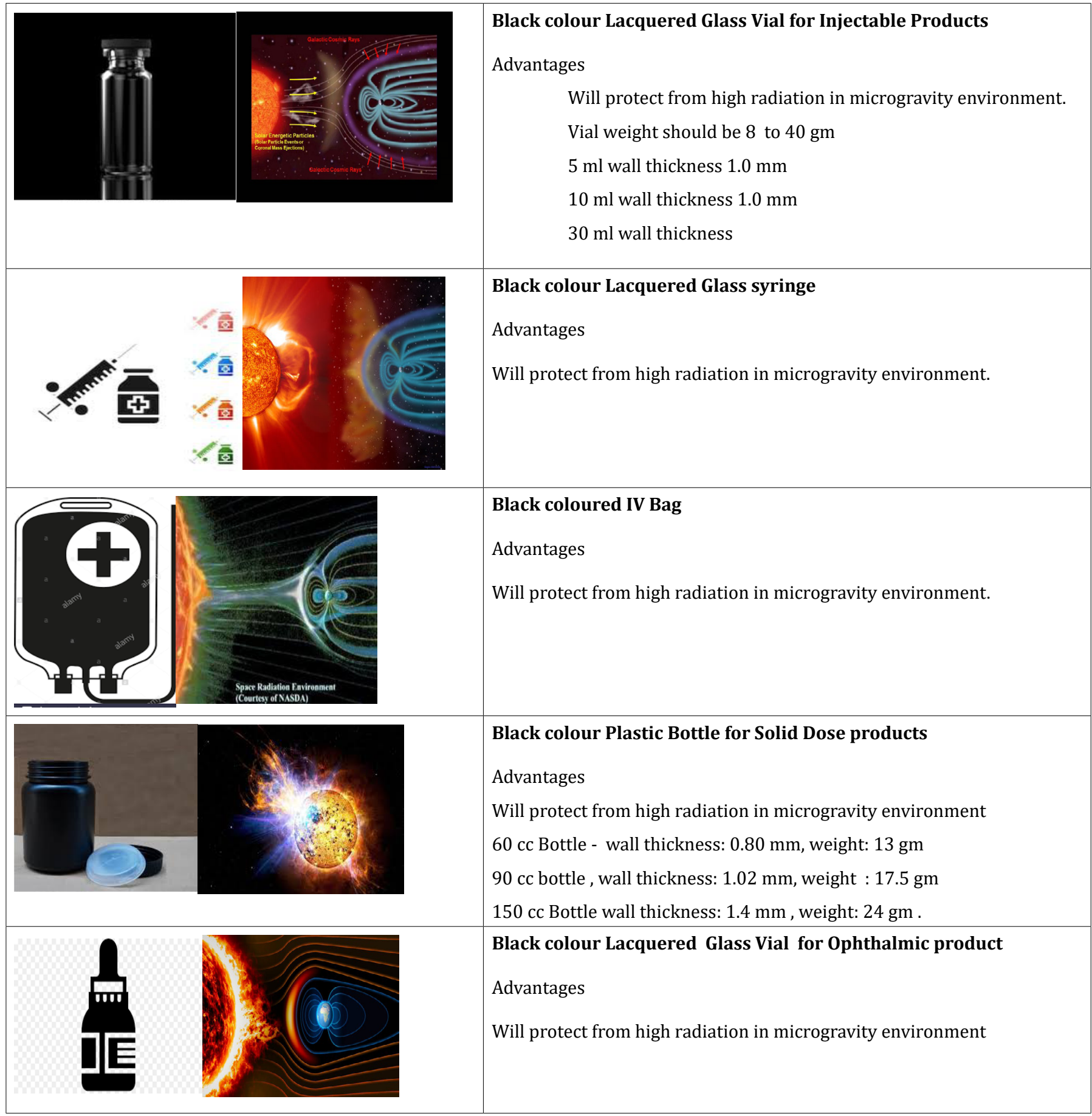

Table 2

Biosimilar product effected - Temperature VS Pressure

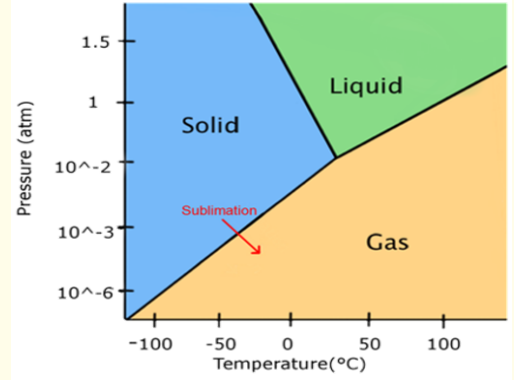

Figure 2

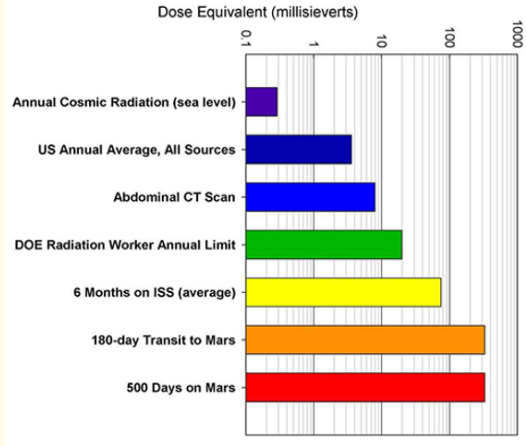

Figure 3 


\section{Radiation tracker in space}

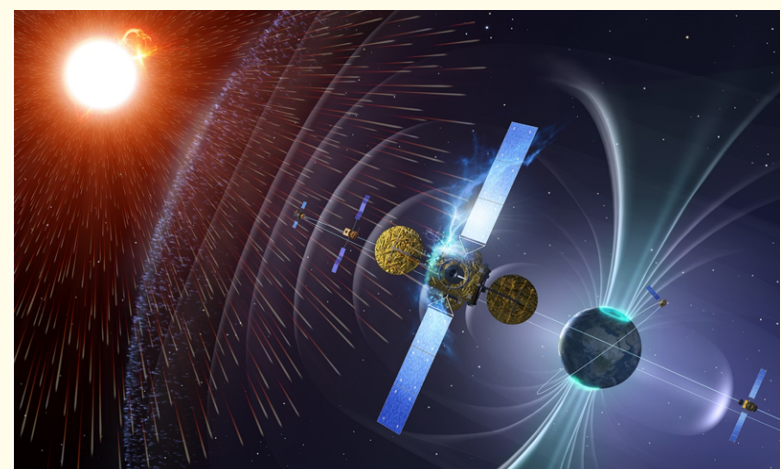

Figure 4

\section{Different types of radiation in space}

- Electromagnetic radiation: Like radio waves, microwaves, infrared, visible light, ultraviolet, $\mathrm{x}$-rays, and gamma radiation $(\gamma)$.

- Particle radiation: Like alpha radiation $(\alpha)$, beta radiation $(\beta)$, and neutron radiation (particles of non-zero rest energy).

- Acoustic radiation: Like ultrasound, sound, and seismic waves (dependent on a physical transmission medium).

- Gravitational radiation: Form of gravitational waves, or ripples in the curvature of space time.

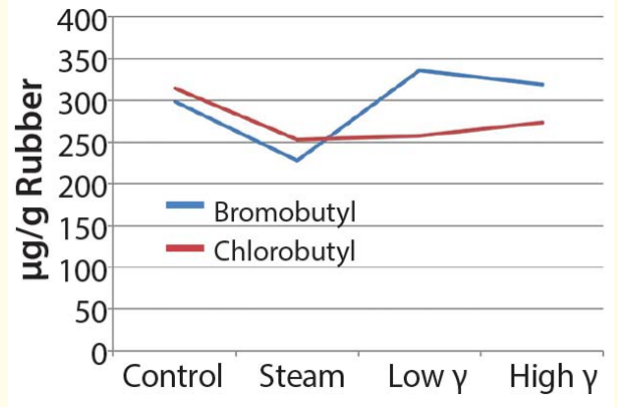

Figure 5

Impact of radiation in plunger

Table 3

Why impurities in product after gamma radiation

After applying Gamma Radiation irreversible changes observed in physical properties of the polymer. Part of these changes are attributed to the chain scission of polymers due to irradiation, crosslinking, breaking of some covalent bonds, and formation of carbon clusters or even libration of free radicals that may also induced the formation of new chemical bonds. During the gamma-irradiated polymers, the release of volatile species almost takes place [1-3].

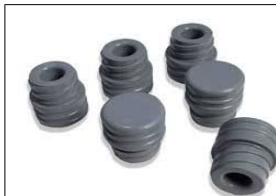

This plunger was exposed by elastomeric materials and molded syringe pistons to either gamma irradiation emitted by cobalt 60 or steam autoclave before evaluation

Conducted tests before and after sterilization of brombutyl and chlorobutyl at 0,6 , and 12 months on the materials' physical and chemical properties.

We conducted chemical tests on duplicate extracts of the test plates using 2-propanol (IPA). We cut approximately $15 \mathrm{~g}$ of each test plate into pieces and refluxed those pieces for four hours. Aggressive conditions were intended to reveal the presence of antioxidants and cure by-products for each elastomeric material. We analyzed the resulting qualitative screening extracts using gas chromatography with a mass-selective detector (GC/MS). Solvent loss was adjusted after reflux to original $50-\mathrm{mL}$ volume to estimate the concentration of significant peaks based on the response of an external standard. A peak was considered for evaluation if the estimated concentration was equal to $0.5 \mu \mathrm{g} / \mathrm{g}$ of sample based on response of a surrogate standard.

We investigated two groups of like compounds:

2,6-di-tert-butylphenol (BHT) and two of its detected transformation/breakdown products hydrocarbons and oligomers.

The compounds in each group were summed and the totals compared for differences. These results indicate the influence of sterilization within a given batch.

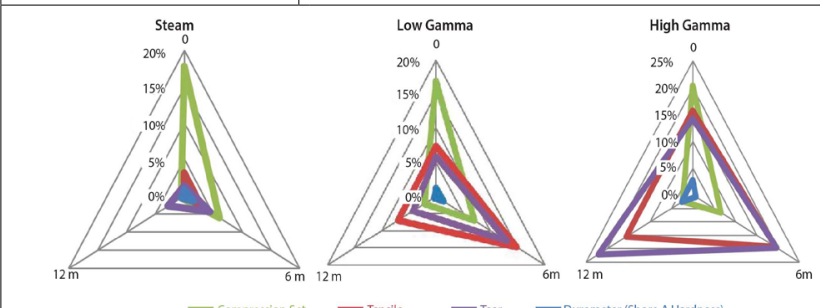

Chlorobutyl percentage difference unprocessed at each sterilization mode

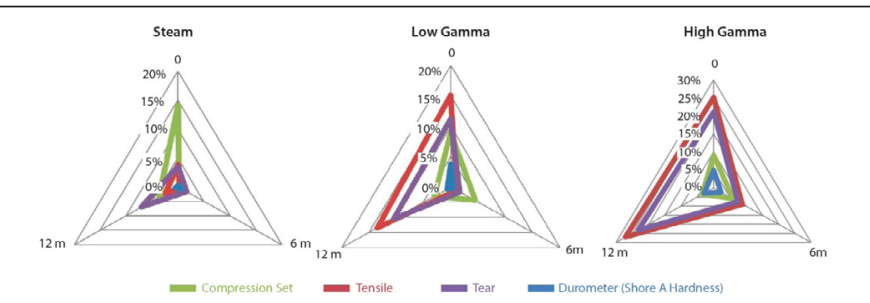

Bromobutyl percentage difference unprocessed at each sterilization mode 


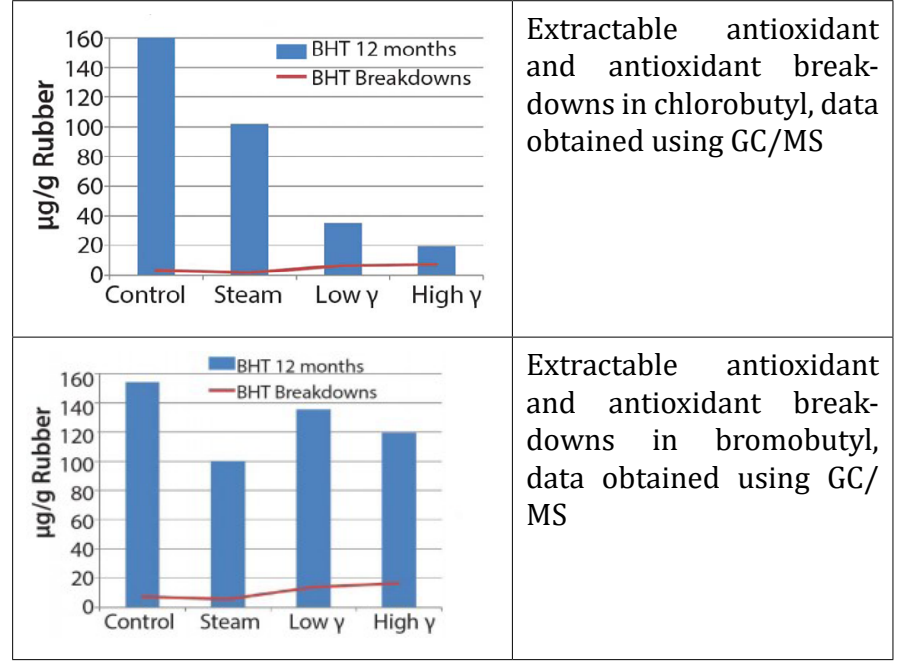

Table 3

How carbon chain changed during gamma radiation

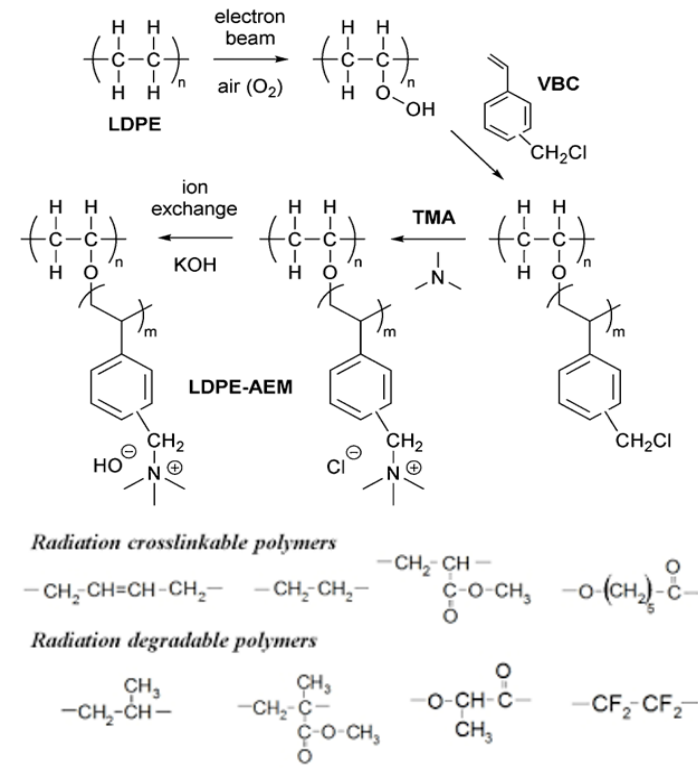

Figure 6

Volume 3 Issue 12 December 2019

(C) All rights are reserved by Anupam Chanda. 